\title{
An Effective Audit Services for Allocation of Data in Cloud
}

\author{
M. Praveen ${ }^{1}$, S. Surya ${ }^{2}$, M. Vadivel ${ }^{3}$, R. Vengatesh ${ }^{4}$ \\ UG Scholar, Department of CSE, M.K.C.E College of Engineering, Karur, India ${ }^{1,2,3,4}$
}

\begin{abstract}
We have created a brand new manner for the reversible information access theme for the multiple authority cloud storage theme. A theme known as CP-ABE is employed as a principle for our project. it's accustomed guarantee each forward and backward security. The results obtained from the project states that the project may be a secure oracle model. It's an internet primarily based application mistreatment MVC design because the principle.
\end{abstract}

Keywords: CP-ABE, Multiple Authority, MVC Design.

\section{INTRODUCTION}

The current propelled technique to personal the information brings regarding the actual security issues. since the cloud servers are not dependable to the general populace. cp-abe is taken into consideration due to the fact the pleasant declare the safety problems by way of giving get admission to rights to the understanding proprietors. $\mathrm{cp}$-abe offers an impact units to function administration and key conveyance. there square degree two varieties of cp-abe frameworks: unmarried-professional cp-abe wherein all houses square measure overseen by means of one professional, and multi-professional cp-abe where traits rectangular measure from absolutely sudden regions and oversaw by way of varied professionals. multiprofessional cp-abe is similarly ok for statistics get to management of disbursed storage

\section{OBJECTIVE}

In multi-specialist disbursed storage frameworks, clients' trends is changed powerfully. a consumer ought to also be entitled a few new residences or denied some present traits. additionally, his consent of statistics get to must be strained to be modified in a while. be that due to the fact it can, present characteristic resignation strategies either be given a conscious server or absence of productiveness, they do now not seem to be quality for addressing the usual denial recede in facts get to administration in multispecialist distributed storage frameworks.

\section{PROBLEM DEFINITION}

In logical educate get to management systems procedures, the knowledge proceeds untrusted functionality is encoded and on these lines the corresponding mystery composing keys secrecy of records is ensured towards the untrusted reposition else as unapproved clients. Appropriations at interims the dispended storage settings. Such a framework is utilised to catch upon absence of qualified employees and best foundation in rustic Asian kingdom. The device projected here is an clever tablet,that is suited instructing completely exceptional dialects. We have a tendency to advice to shape initiative instructive programming which would possibly preserve running at the pill. The products enables the purchaser to work out a way to compose and more over spell the letter sets. First of all projects show letters in order after which moves onto words and sentences.

\section{IMPLEMENTATION}

The software depends at the three degree MVC design. With the aid of using this software it is created to make up an software inside the cloud. On those strains creating the information the expertise information in the cloud to land up virtually more secure than the opposite statistics. It is created by means of cryptography the expertise before golf stroke away it in the cloud. Patron or the statistics information on the records businessman is responsible of planning to the understanding in the cloud and what's greater to relinquish protection to his own information.

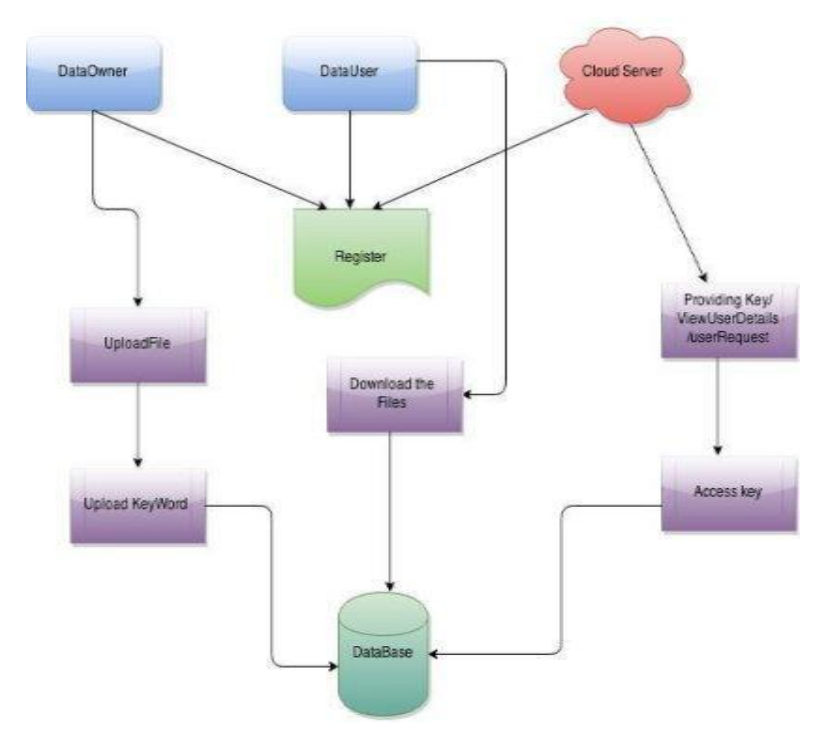

Figure 1: System Architecture 
Therefore through cryptography the expertise before golfing stroke away the expertise in the cloud he created the knowledge to be cosy. On the off possibility that if the knowledge is taken by unapproved people then he could not have the functionality to examine the expertise because it's far disorganised. Increase mystery writing of the know-how is created by way of the cloud specialist coop. the important thing turned into given to the expertise businessman to the information. Therefore at some stage in this indicates there square measure 2 secret writing of the know-how. Therefore its created cosy to the expertise

\section{RELATED WORKS}

\section{A Literature Survey On Scalable And Effective Audit Services For Storages Data In Clouds}

Author: V. Goyal, A. Jain,O. Pandey, and A. Sahai

Comparison of secret key and public key primarily based 3-DES and RSA algorithms is completed. Therefore 3DES is employed. RSA and 3- DES take issue from one another in sure options. In cryptography 3-DES is healthier than all different algorithms in turnout and power consumption. the chosen algorithms 3-DES and RSA square measure mentioned in cloud setting. However RSA consumes Brobdingnagian amount of your time to realize encoding and cryptography method. Comparison result showed that 3-DES has higher performance than RSA. From the Comparison results, I evaluated that turnout of 3DES algorithmic program is far higher than the turnout of RSA algorithmic program. we tend to style AN communicatory, economical and rescindable knowledge access management theme for multiauthority fog loading schemes, wherever there square measure numerous establishments co-exist and every expert is equipped to subject qualities severally. Unambiguously, we have a habit of near suggest a adjustable theme and relate it because the primary methods to flair the data admittance running theme. We tend to initial propose a rescindable multiauthority $\mathrm{CP}-\mathrm{ABE}$ theme, wherever AN economical and secure scheme is projected to unravel the attribute revocation downside within the system. As a consequence of the important modernize is applied by every feature specialist not the waiter.

Scalable and Secure Sharing Of Personal Health Records In Cloud Computing Using Attribute - Based Encryption

Author: M. Li, S. Yu, Y. Zheng, K. Ren,

Our projected system perform more than one understanding get entry to management in Cloud Computing. we generally tend to get approach in information proprietor/facts consumer/Cloud Server. Transferred report's in information owner method to be tired automatic inscribe and Lock the document and get into the Cloud Admin, Cloud Admin consists presenting Keys, examine client info and consumer Request for top Key and click on on supplier Key to planning to Key for expertise proprietor. facts owner includes transfer information in Encrypted layout and switch wanting list in
Encrypted layout and study Request Permission for get to the record. a statisticsinformationan facts shopper method downloaded unique information and enter into top keyword and send the key Request to the cloud Server. A cloud Server given Permission Granted for Key to transfer the document and specific key ship to appropriate knowhow user Mail identity and discover Authority from requested to CA and Granted to result in to Cloud Server.

As a great prevalence, a department of Veterans Affairs know-how containing sensitive letter of twenty six.5 million military veterans, and conjointly their social protection numbers relate degreed scientific issues become taken by way of a operating man international health organisation took the information domestic while now not approval. to ensure quiet driven safety management over their very own specific PHRs, it is fundamental to own satisfactory-grained records get to management elements that job with semi-confided in servers. A conceivable and promising method is write in code the facts earlier than outsourcing. commonly, the PHR businessman herself ought to choose associate degree method to write in code her facts and to permit that arrangement of purchasers to access every file. A PHR report must to completely be out there to the consumers global health organisation area unit given the proportionate cryptography critical, but stay trusty to the accumulation of consumers.

\section{EXISTING SYSTEM}

In the existing system the cloud is providing security to the data by encrypting the data in the cloud

\section{DISADVANTAGES}

Thus in the existing the user does not have the access to authorize the data. The security is only provided by the cloud service providers.

\section{PROPOSED SYSTEM}

Our proposed System Perform Multiple Data Access Control in Cloud Computing. We get Process in Data Owner/Data User/Cloud Server. Uploaded File's in Data Owner process to be done in Automatic Encrypt and Lock the file and enter into the Cloud Admin, Cloud Admin Consists Providing Keys, View user Details and User Request for Top Key and Click Provider Key to accessing Key for Data Owner. Data Owner Consists of upload data in Encrypted Format and upload searching index in Encrypted Format and View Request Permission for access the file.

A Data user Process downloaded Particular data and Enter into Top Keyword and send the key Request to the cloud Server. A cloud Server given Permission Granted for Key to Download the File and Particular key send to Appropriate Data User Mail Id and Get Authority from Requested to CA and Granted to access Cloud Server 


\section{MODULES}

\section{Data owner}

Data owner is the module in which the data is owned by the user. Thus by owning the authorization of the data the user can have the entire access to it. Thus the data is made secured by the user itself. Thereby enhancing the security of the data.

\section{Provider}

Provider is the module in which cloud service provider comes to the point that it can have the entire access to the data thus by providing the data service point to the user. The data can have the access control owned by the user although but it is made it clear by the way.

\section{User}

In this module, Users unit having confirmation and security to get to the detail that is given inside the reasoning framework.

Before getting to or attempting the modest print client got the chance to have the record amid this else they got the chance to enroll beginning client can enlist their points of interest like client name, secret key, email, versatile no, and after that. we tend to have a tendency to build up this module, where the distributed storage is made secure.

\section{Security Model}

In this module, customers unit having approval and safety to get to the element this is given within the concept system.

Earlier than attending to or attempting the unassuming print supporter got the risk to have the report within the midst of this else they were given the risk to enroll early on customer can be a part of their factors of intrigue like benefactor call, thriller specific, e mail, adaptable no, and after that. we have a tendency to will be predisposed to accumulate this module, wherein the administered carport is made secure.

\section{Multiple-Authority}

It is the one in which the access control rights have the certain the certain feature that it can be formatted thus it can be consolidated the paper.

\section{() Data Encryption by Owners}

(B) Data Decryption by Users

\section{Data Encryption by Owners:}

It is the one in which the data owner is the is the one which is made entirely clear about the decision in which the security is made by the user.

\section{Data Decryption by Users:}

It is the one in which the encrypted data is decrypted by the user which can have the full access control over the data.

\section{CONCLUSION}

Thus in this paper we have granted the fullest security to the data. Thus by enhancing the security to the data. Thus the user first encrypts the data by their own and also the data is made entirely secure by the user itself. Thus before storing the data in the cloud the user himself self-encrypt the data and then he stores the data. Thus the data can also be encrypted again by the cloud service providers. Thus the data is made secure to the fullest extent to the data by the security to the data. Thus it is made clear the data stored in the cloud is made secure.

\section{REFERENCES}

[1] S.Saravanan, Arivarasan."An efficient ranked keyword search for effective utilization of outsourced cloud data" Journal of Global Research in Computer Science, Vol4(4), pp:8-12

[2] S Saravanan, V Venkatachalam ,“ Improving map reduce task scheduling and micro-partitioning mechanism for mobile cloud multimedia services" International Journal of Advanced Intelligence Paradigms ,Vol 8(2),pp157- 167,2016.

[3] S Saravanan, V Venkatachalam ,“ Advance Map Reduce Task Scheduling algorithm using mobile cloud multimedia services architecture" IEEE Digital Explore,pp21-25,2014.

[4] S Saravanan, V Venkatachalam ,"Enhanced bosa for implementing map reduce task scheduling algorithm" International Journal of Applied Engineering Research,Vol 10(85),pp60-65,2015.

[5] Karthika, K. P. Porkodi ,"FRAUD CLAIM DETECTION USING SPARK" International Journal Of Innovations In Engineering Research And Technology ISSN: 2394-3696 VOLUME 4, ISSUE 2, Feb.-2017

[6] I.Karthika, K.P.Porkodi," AUTOMATIC MONITORING AND CONTROLLING OF WEATHER CONDITION USING BIG DATA ANALYTICS , International Journal of Advanced Research in Computer and Communication Engineering Vol. 6, Issue 1, January 2017

[7] Karthika, P. Gokulraj, S. Saravanan" Prediction of sales using Big data analytics" Journal Of Advances In Chemistry Vol 12, No 20

[8] Karthika, S. Priyadharshini " Survey on Location based sentiment analysis of Twitter data" (C) 2017 IJEDR | Volume 5, Issue 1 | ISSN: 2321-9939

[9] M. Chase, "Multi-Authority Attribute Based Encryption," in Proc. 4th Theory of Cryptography Conf. Theory of Cryptography (TCC'07), 2007, pp. 515-534.

[10] M. Chase and S.S.M. Chow, "Improving Privacy and Security in Multi-Authority Attribute-Based Encryption," in Proc. $16^{\text {th }}$ ACM Conf. Computer and Comm. Security (CCS'09), 2009, pp. 121-130. 